\title{
Fibroblast Growth Factor and Glutamate: Opposing Roles in the Generation and Degeneration of Hippocampal Neuroarchitecture
}

\author{
Mark P. Mattson, ${ }^{1}$ M. Murrain, ${ }^{2}$ P. B. Guthrie, ${ }^{2}$ and S. B. Kater ${ }^{2}$ \\ 'Sanders-Brown Center on Aging, Department of Anatomy and Neurobiology, University of Kentucky Medical Center, \\ Lexington, Kentucky 40536, 2Program in Neuronal Growth and Development, Department of Anatomy and Neurobiology, \\ Colorado State University, Fort Collins, Colorado 80523
}

Neuritic regression and cell death (neurodegeneration) are common features of both normal nervous system development and neurodegenerative disorders. Growth factors and excitatory amino acid neurotransmitters have been suggested independently to play roles in neurodegenerative processes. The present study investigated the combined effects of fibroblast growth factor (FGF) and glutamate on the development and degeneration of cultured hippocampal neurons. Consistent with previous data, we found that FGF, but not NGF, promoted neuronal survival and dendritic outgrowth. In contrast, a low level of glutamate (50 $\mu \mathrm{M})$ caused a reduction in dendritic outgrowth, and high levels (100 $\mu \mathrm{M}-$ $1 \mathrm{~mm}$ ) reduced neuronal survival in a dose-dependent manner. When cultures were maintained in the presence of FGF, there was a striking reduction in neuronal death normally caused by 100-500 $\mu \mathrm{m}$ glutamate. FGF raised the threshold for glutamate neurotoxicity. FGF also antagonized the outgrowth-inhibiting actions of glutamate. Measurements of intracellular calcium levels with fura-2 demonstrated a direct relationship between glutamate-induced rises in intracellular calcium and neurodegeneration. FGF reduced the glutamate-induced increases in intracellular calcium levels. However, when cultures were pretreated with the RNA synthesis inhibitor actinomycin D or with the protein synthesis inhibitor cycloheximide, FGF did not prevent glutamate-induced increases in intracellular calcium or neurodegeneration. Taken together, these results suggest that (1) interactions between growth factors and neurotransmitters may be important in brain development; (2) imbalances in these systems may lead to neurodegeneration; and (3) cellular calclum-regulating systems may be a common focus of growth factor and neurotransmitter actions.

Within the nervous system, individual neurons are exposed to many environmental signals which influence their form and function. Prominent among these signals are growth factors and neurotransmitters. Many studies have investigated roles for individual growth factors (see Levi-Montalcini, 1976; Greene and Shooter, 1980; Varon et al., 1988, for reviews) or neurotrans-

\footnotetext{
Received Feb. 3, 1989; revised Apr. 13, 1989; accepted Apr. 19, 1989.

We thank B. Bertram, D. Giddings, and B. Hayes for technical assistance. Supported by NIH grants NS 08054, NS2456I, and NS15350 and a fellowship to M.P.M. from the John Douglas French Foundation for Alzheimer's disease.

Correspondence should be addressed to Dr. Mark P. Mattson, 211 SandersBrown Building, University of Kentucky Medical Center, Lexington, KY 405360230.

Copyright $\odot 1989$ Society for Neuroscience $0270-6474 / 89 / 113728-13 \$ 02.00 / 0$
}

mitters (see Lauder, 1988; Mattson, 1988; Lipton and Kater, 1989 , for reviews) in neuronal development and plasticity. In addition to their involvement in development and plasticity, growth factors and neurotransmitters have each been implicated in the abnormal neurodegeneration associated with CNS disorders such as parkinsonism, Huntington's disease, and Alzheimer's disease (Schwarcz et al., 1984; Appel, 1986; Choi, 1988; Mattson, 1988). Since it is clear that neither individual growth factors nor individual neurotransmitters act in isolation in the intact nervous system, we tested the hypothesis that neurotransmitters and growth factors can interact to regulate cell survival and neurite outgrowth.

Growth factors act as trophic and neurite outgrowth-promoting signals during neuronal development (Cowan et al., 1984; Black, 1986; Purves, 1986; Varon et al., 1988). The actions of NGF on vertebrate sensory and sympathetic neurons have been well-characterized (Levi-Montalcini, 1976; Greene and Shooter, 1980). Recently, considerable attention has focused on putative growth factors for CNS neurons (Korsching, 1986; Fine and Rubin, 1988). For example, NGF and NGF mRNA are expressed at quite high levels in the hippocampus (Shelton and Reichardt, 1986; Whittemore et al., 1988), NGF infusion prevents the loss of basal forebrain cholinergic markers in septumlesioned rats (Hefti et al., 1984), and basal forebrain cholinergic cells express the NGF receptors at high levels (Kordower et al., 1988). Additional hippocampal-derived trophic factors, such as a recently described central cholinergic trophic factor, may play roles in the maintenance of basal forebrain/septum cholinergic neurons (Bostwick et al., 1987). Furthermore, growth factors previously known for their actions on non-neuronal cells may also influence neuronal survival and outgrowth in the CNS. For example, fibroblast growth factor (FGF) is produced at high levels in the developing rat brain, levels decrease with maturation (Gospodarowicz et al., 1986; Caday et al., 1988), and the expression of FGF mRNA increases in lesioned rat brain (Logan, 1988). FGF has also been shown to promote the survival of cultured cerebral cortical (Morrison et al., 1986; Unsicker et al., 1987; Hatten et al., 1988) and hippocampal (Walicke et al., 1986; Necdcls and Cotman, 1988) neurons. Taken together, these data are consistent with roles for growth factors in CNS development. In addition to developmental roles, growth factors may also be important in adult plasticity and in aging and disease. Data suggest that growth factors such as NGF may be involved in adaptive mechanisms, such as sprouting, that occur in response to alterations in activity or injury in the adult CNS (Crutcher, 1987). Roles for growth factors in age-related neuronal losses and in specific neurodegenerative disorders, in- 
cluding Alzheimer's disease, parkinsonism, and amyotrophic lateral sclerosis, have also been suggested (Appel, 1986; Korsching, 1986; Fine and Rubin, 1988).

Neurotransmitters constitute another class of intercellular signaling molecules which may play important roles in neuronal development and degeneration. Data obtained in a number of neural systems suggests strongly that neurotransmitters have developmental roles as regulators of neurite outgrowth and synaptogenesis. For example, growth cone motility in specific identified molluscan neurons is inhibited by serotonin (5-HT) or dopamine (Haydon et al., 1984; McCobb et al., 1987); ACh (Lipton et al., 1988) and dopamine (Lankford et al., 1987) inhibit neurite outgrowth in chick retinal neurons; opioids may suppress dendritic outgrowth in several regions of the mammalian brain (Hauser et al., 1987); glutamate selectively inhibits dendritic outgrowth in isolated hippocampal pyramidal-like neurons (Mattson et al., 1988a); glutamate has trophic effects in cerebellar development (Pearce et al., 1987; Balazs et al., 1988). Synapse formation and plasticity may also be subject to regulation by neurotransmitters. Recent cell culture studies demonstrated that glutamate, released from entorhinal axons, can promote the formation of synapses with target hippocampal pyramidal neurons (Mattson et al., 1988b). In situ studies demonstrated that blockade of NMDA receptors in the vertebrate visual system results in a disruption of the normal segregation of synapses in the tectum (Cline et al., 1987). In addition, there is very strong evidence that glutamate mediates the synaptic plasticity associated with long-term potentiation in the mammalian hippocampus (Chang and Greenough, 1983; Desmond and Levy, 1983; Lynch, 1986). Finally, imbalances in neurotransmitter systems may result in neurodegencration (scc Choi, 1988; Mattson, 1988, for reviews). Indeed, there is now convincing data showing that glutamate is involved in the neuronal degeneration that occurs in the hippocampus in stroke and epilepsy (Sloviter, 1983; Schwarcz et al., 1984; Simon et al., 1984; Rothman and Olney, 1986; Choi, 1988). Glutamate may also play a role in the neurodegeneration that occurs in Alzheimer's and Huntington's diseases (Coyle and Schwarcz, 1976; Maragos et al., 1987; Mattson, 1988).

The first evidence that different neurite outgrowth-regulating signals can interact on the same neuron came from recent cell culture studies. McCobb et al. (1988) found that ACh prevented 5-HT-induced inhibition of growth cones in identified snail neurons. Mattson and Kater (1989a) demonstrated that activation of GABA receptors reduces glutamate-induced dendrite regression in hippocampal pyramidal neurons. The latter study also demonstrated that GABA can reduce glutamate neurotoxicity. These findings suggested that relative levels of input from different neurotransmitters can determine whether a neuron is present and whether its outgrowth status is in a progressive or regressive mode. In the present study, we extend these initial findings and test the hypothesis that neurotransmitters and growth factors can interact to regulate neuroarchitecture.

The cellular mechanisms by which growth factors and neurotransmitters influence neurite outgrowth and cell survival are not yet clear. The survival-promoting effects of NGF apparently require its retrograde transport from axonal endings to the cell soma, and protein synthesis inhibitors prevent the outgrowthpromoting effects of NGF (Mizel and Bamburg, 1976). These data are consistent with the hypothesis that NGF influences the expression of mRNAs and proteins which promote neuronal survival. The mechanisms by which growth factors affect neurite outgrowth are likely to be somewhat different than the trophic mechanisms since growth factors such as NGF can exert effects locally at the growth cone (Gundersen and Barrett, 1980). Intracellular messenger systems, including cAMP and calcium, may be involved in the outgrowth-promoting actions of NGF (Schubert et al., 1978; Varon et al., 1988). In the case of neurotransmitter actions on neurite outgrowth and cell survival, convincing cvidence obtaincd from a varicty of neural systems indicates that intracellular calcium is involved mechanistically (Cohan et al., 1987; Mattson and Kater, 1987; Choi, 1988; Kater et al., 1988; Mattson, 1988). In hippocampal pyramidal neurons, low levels of glutamate selectively inhibit dendritic outgrowth, an effect mediated by calcium influx through plasma membrane channels (Mattson et al., 1988a, c, 1989). Glutamate and related excitatory amino acids can also induce large, sustained rises in intracellular calcium levels (Connor et al., 1988; Mattson et al., 1988d, 1989), which are causally linked to cell death (Choi, 1988; Mattson et al., 1988a, d, 1989; Siesjo, 1988). Such alterations in neuronal calcium-regulating systems have been suggested to contribute to neurodegeneration in several disorders (Choi, 1988; Kater et al., 1989; Mattson, 1989).

It is of considerable importance, in the context of the intact nervous system, to understand how different environmental signals intcract to yicld a net effect on neuroarchitecture. We have therefore employed a tractable hippocampal culture model of neuronal development and degeneration to address this problem. Our data demonstrate that growth factors and neurotransmitters can have opposing effects in the development and degeneration of neuroarchitecture. These findings, along with other studies, suggest that these 2 types of signals can interact in neuronal development and that imbalances in these systems may lead to neurodegeneration in aging and disease.

\section{Materials and Methods}

Cell cultures. Dissociated cell cultures of embryonic (E18) rat hippocampus were established and maintained from cryopreserved stocks as described previously (Mattson and Kater, 1988). Dissociated cells were seeded at low density (approximately 50 cells $/ \mathrm{mm}^{2}$ of culture surface) in culture dishes with a polylysine-coated plastic substrate (for fura-2 experiments, the culture dishes had polylysine-coated glass coverslips affixed to their bottoms; Mattson et al., 1989) and containing $2 \mathrm{ml}$ of $\mathrm{MEM}+$ medium. MEM + medium consisted of Eagle's minimum essential medium (Gibco) supplemented with $2 \mathrm{~mm}$ L-glutamine, $10 \mathrm{~mm}$ $\mathrm{Na}_{2} \mathrm{HCO}_{3}, 2 \%$ glucose, $15 \mathrm{~mm} \mathrm{KCl}, 1 \mathrm{~mm}$ sodium pyruvate, and $10 \%$ (vol/vol) fetal bovine serum. Following a $4 \mathrm{hr}$ incubation in a $95 \%$ air/ $5 \% \mathrm{CO}_{2}$ humidifed incubator $\left(36^{\circ} \mathrm{C}\right)$, the culture medium was replaced with $1.5 \mathrm{ml}$ of MEM+ medium containing 10\% Nu-Serum (Collaborative Research, Bedford, MA) in place of the fetal bovine serum. In a previous study we found that reduction of the medium volume to approximately $0.7 \mathrm{ml}$ promoted long-term neuronal survival (Mattson and Kater, 1988). In the present study, we maintained cultures in a larger $(1.5 \mathrm{ml})$ medium volume which was suboptimal for survival in order to accentuate positive effects of growth factors on cell survival. Cultures were maintained at $36^{\circ} \mathrm{C}$ in a $95 \%$ air $/ 5 \% \mathrm{CO}_{2}$ humidified incubator. Glial cells are very sparse in cultures established and maintained under the conditions just described and constitute less than $2 \%$ of the cells. Two major factors contributing to the relative lack of glia are that gliosis is only in the very early stages in E18 embryos, and the few glia that do enter the cultures divide quite slowly in the low-density cultures. This paucity of glial cells provided the opportunity to directly assess the effects of glutamate and growth factors on neuronal cells without complications in interpretation that might otherwise arise in cultures containing large numbers of contaminating glia.

Assessments of neurite outgrowth and cell survival. Cultures were examined and photographed using a Nikon Diaphot inverted microscope with phase-contrast optics. Measurements of neurite outgrowth were 


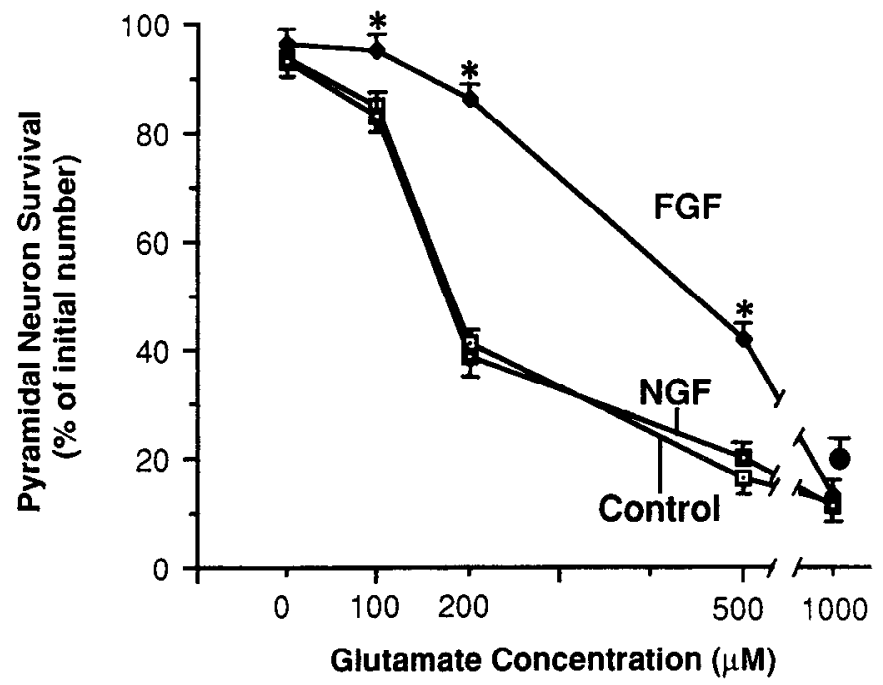

Figure 1. FGF protects against glutamate neurotoxicity. In control cultures, maintained in the absence of growth factors, glutamate caused a dose-dependent reduction in pyramidal neuron survival (glutamate was added on culture day 5 and cell survival was determined $24 \mathrm{hr}$ later). In parallel cultures maintained in the presence of NGF (final concentration of $10 \mathrm{ng} / \mathrm{ml}$ added on days 0,2 , and 4), glutamate also caused quantitatively similar levels of cell death. FGF $(5 \mathrm{ng} / \mathrm{ml}$ final concentration, added on culture days 0,2 , and 4) reduced glutamateinduced neurodegeneration significantly in cultures exposed to 100,200 , or $500 \mu \mathrm{M}$ glutamate $\left({ }^{*} p<0.05-0.001\right)$. Closed circle, $20 \mathrm{ng} / \mathrm{ml}$ FGF plus $1 \mathrm{mM}$ glutamate. Values represent means and SEM $(n=8$ fields in 2 separate cultures).

made from tracings of projected photographic negatives of pyramidallike neurons selected randomly on culture day 5 (cf. Figs. 4, 5). Pyramidal-like neurons were identified by morphological criteria; these neurons have one major axon-like process and several minor dendrite-like processes (Dotti et al., 1988; Mattson et al., 1988a). Numbers of primary dendrites and branch points/neurite were simply counted. Neurite lengths were determined using the calibration provided by a photographic negative of a microscope stage micrometer. Cell survival was assessed by examining marked microscope fields at successive time points as described previously (Mattson et al., 1988a); at least 3 fields in 2 separate dishes were examined. Surviving neurons were those that were still present and met the established morphological criteria of viable neurons (Mattson et al., 1988a). Survival rates were expressed as a percentage of the initial number of pyramidal-like neurons in the marked fields. Experiments which tested the effects of glutamate on cell survival were carried out on culture days 5 or 6 . Since neuronal sensitivity to the outgrowth-regulating and degenerative actions of excitatory amino acids increases with time in culture (Mattson et al., 1988a; Mattson and Kater, 1988), it was important to conduct all experiments in cultures at a similar stage of development. Counts of viable neurons were made immediately before and $24 \mathrm{hr}$ after exposure to glutamate. Unless otherwise stated, growth factors were added to the culture medium on culture day 0 and on every other day thereafter. Experimental agents and sources were as follows: FGF, Collaborative Research (CR-FGF, contains both basic and acidic forms of FGF); NGF, Chemicon (7S); L-glutamate, actinomycin D, and cycloheximide, Sigma. All test agents were prepared as stocks in culture medium and were added to cultures in 5-200 $\mu \mathrm{l}$ volumes.
Fura-2 measurements of intracellular calcium levels. Details of the fura-2 methods are reported elsewhere (Mattson et al., 1988d, 1989). Briefly, cultures were exposed to fura-2/AM (Molecular Probes, Eugene, OR; $2 \mathrm{~mm}$ in DMSO diluted to a final incubation concentration of 6 $\mu \mathrm{M})$ for $30 \mathrm{~min}$, washed, and incubated for $60 \mathrm{~min}$ to allow hydrolysis of the ester. Using this method of loading results in the presence of fura-2 in subcellular vesicular compartments as well as in the cytoplasm. Therefore, measurements represent a weighted average of intracellular free calcium levels. The cultures were viewed on a Zeiss ICM microscope with a Quantex CCD, low-light camera; the camera output was fed to a Quantex (Santa Clara, CA) QX7-210 image-processing system, where it was converted to a $640 \times 480$ digital image ( 256 gray levels) and averaged for $540 \mathrm{msec}$ (16 frames). The excitation wavelength was determined by a computer-controlled filter wheel which rotated between $350 \pm 10$ and $380 \pm 10 \mathrm{~nm}$ interference filters. Neutral density filters $(0.12 \mathrm{ND})$ were inserted in the excitation path to reduce bleaching of the fura- 2 and prevent saturation of the camera by the emission from the ccll body. Becausc of the reduccd cxcitation intensity, the fluorescent signal from the processes was close to background noise level; although measureable ratios could be obtained from the processes, calcium concentrations were taken only from the soma. Measurements at each time point were made in neurons located in different regions of the same culture dish; this procedure reduces any chance of photodamage that can occur with repeated imaging of the same neurons. The fluorescent emission was filtered with a $495 \mathrm{~nm}$ long-pass emission filter. Fluorescent images were captured using each excitation filter; the ratio $(R)$ of fluorescence intensity $(350 \mathrm{~nm}$ image $) /(380 \mathrm{~nm}$ images) was converted to calcium concentration using the formula [Ca] $=K_{d}\left[\left(R-R_{\min }\right) /\left(R_{\max }\right.\right.$ $-R)]\left(F_{0} / F_{s}\right)$ (Grynkiewicz et al., 1985). For our system, $R_{\min }=0.48$, $R_{\max }=11 ;\left(F_{0} / F_{S}\right)=10 ; K_{d}=224$.

\section{RESULTS}

\section{FGF protects against glutamate neurotoxicity}

Glutamate neurotoxicity was reduced significantly when neurons were maintained in the presence of FGF $(5 \mathrm{ng} / \mathrm{ml}$ added on culture days 0,2 , and 4) (Fig. 1). In the absence of FGF, glutamate ( $100 \mu \mathrm{M}-1 \mathrm{mM}$ ) caused significant reductions in pyramidal neuron survival over a $24 \mathrm{hr}$ exposure period (Fig. 1). Approximately $85 \%$ of the pyramidal neurons survived exposure to $100 \mu \mathrm{M}$ glutamate, $40 \%$ survived exposure to $200 \mu \mathrm{M}$ glutamate, and only $15-20 \%$ survived exposure to $500 \mu \mathrm{M}$ or 1 mM glutamate. The most striking protective effect of FGF on glutamate-induced neurotoxicity was seen in cultures exposed to $200 \mu \mathrm{M}$ glutamate, where FGF improved survival by greater than $210 \%$. The region of rescuc of hippocampal neurons by FGF fell within the glutamate concentration range of 100-500 $\mu \mathrm{M}$; FGF at concentrations up to $20 \mathrm{ng} / \mathrm{ml}$ did not reduce neuronal death induced by $1 \mathrm{~mm}$ glutamate (Fig. 1). Thus, FGF raised the threshold for glutamate neurotoxicity. Micrographs of neurons in control and FGF-treated cultures, before and 24 $\mathrm{hr}$ after exposure to glutamate are presented in Figure 2. In one experiment we tested the time dependence of exposure to FGF on protection against glutamate neurotoxicity. Cultures were maintained in the absence of FGF until culture day 5, and then FGF was added to the cultures 2, 12, or $24 \mathrm{hr}$ prior to exposure to $500 \mu \mathrm{M}$ glutamate. Cell survival $24 \mathrm{hr}$ following exposure to glutamate was significantly enhanced in cultures pretreated with FGF for 12 or $24 \mathrm{hr}$ but not in cultures pretreated with FGF

Figure 2. FGF reduces glutamate-induced degeneration. $A$ and $A_{1}$, Micrographs of hippocampal pyramidal neurons maintained in the absence of FGF prior to (left) and $24 \mathrm{hr}$ following (right) exposure to $200 \mu \mathrm{M}$ glutamate. $B$ and $B$, Micrographs of hippocampal pyramidal neurons in a culture maintained in the presence of FGF $(5 \mathrm{ng} / \mathrm{ml}$ final concentration, added on culture days 0,2 , and 4$)$ prior to (left) and $24 \mathrm{hr}$ following (right) exposure to $200 \mu \mathrm{M}$ glutamate. Scale bar, $25 \mu \mathrm{m}$. 

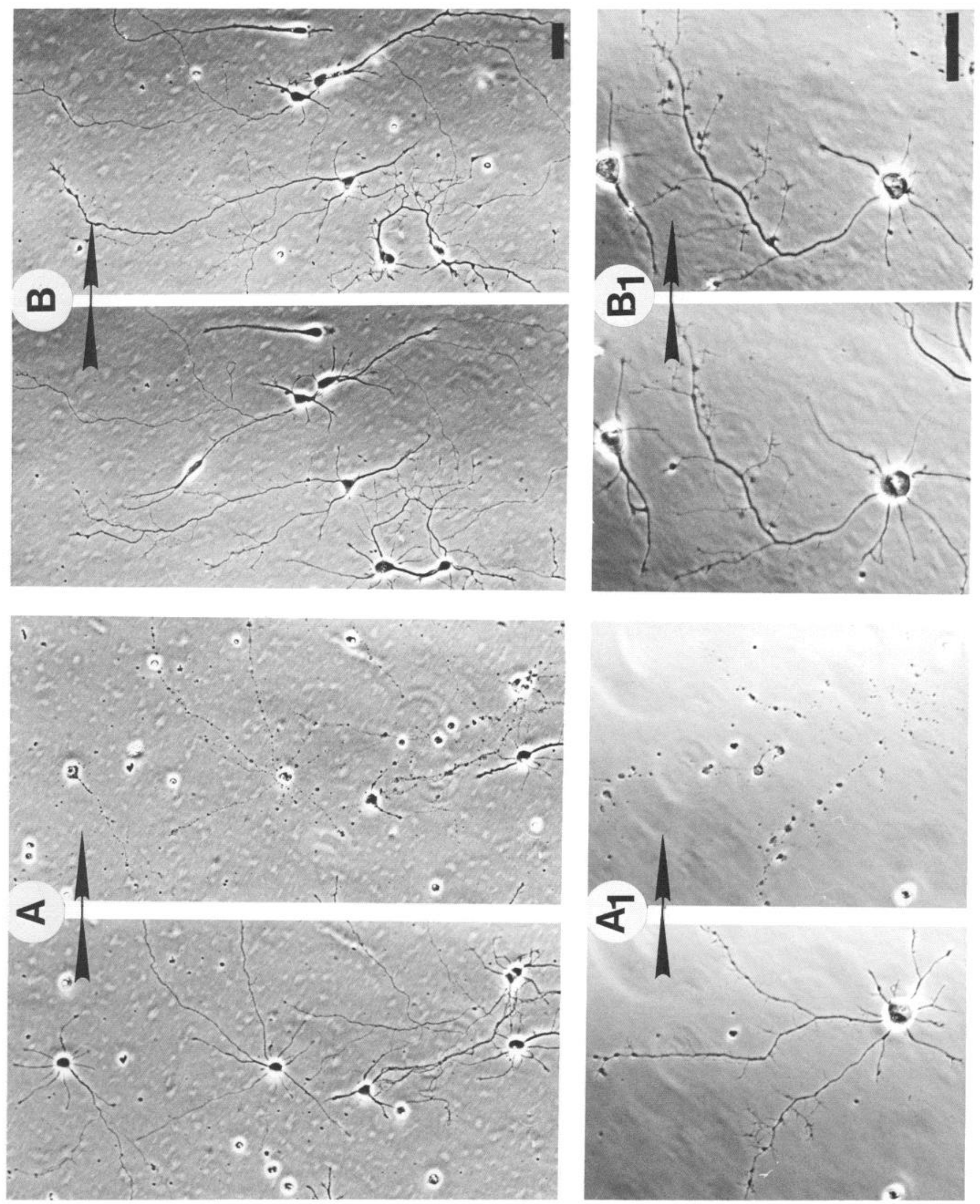


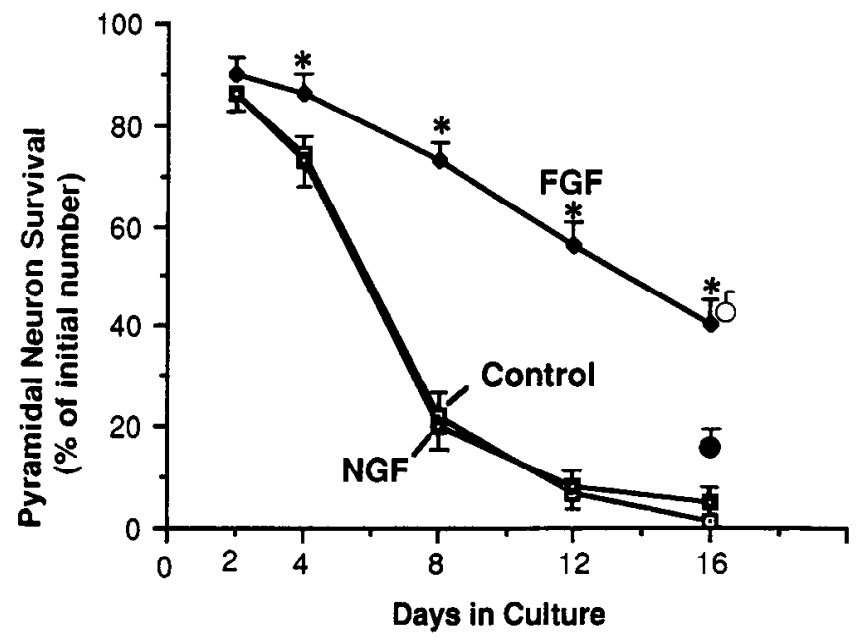

Figure 3. FGF promotes long-term survival of pyramidal neurons. In control cultures, maintained in the absence of growth factors, there was a progressive reduction in pyramidal neuron survival with time in culture such that essentially no viable pyramidal neurons remained by culture day 16. Similar results were seen in cultures maintained in the presence of NGF (final concentration of $10 \mathrm{ng} / \mathrm{ml}$ added on culture day 0 and every other day thereafter). FGF ( $5 \mathrm{ng} / \mathrm{ml}$ final concentration, added on culture day 0 and every other day thereafter) enhanced neuronal survival significantly from culture days $4-16\left({ }^{*} p<0.05-0.001\right)$. The open circle at day 16 represents survival in cultures treated with $10 \mathrm{ng} / \mathrm{ml} \mathrm{FGF}$, and the closed circle represents cultures treated with 1 $\mathrm{ng} / \mathrm{ml}$ FGF (FGF was added on culture day 0 and every other day thereafter. Values represent means and SEM $(n=4-6$ fields in 2 separate cultures).

for $2 \mathrm{hr}$. Survival rates were as follows: control cultures, $16 \pm$ 2.9\%; 2 hr FGF pretreatment, $20 \pm 2.9 \% ; 12$ hr FGF pretreatment, $28 \pm 1.8 \%$ ( $p<0.01 \%$ vs control); 24 hr FGF pretreatment, $39 \pm 2.2 \%$ ( $p<0.001$ vs control). Thus, the ability of FGF to protect against glutamate neurotoxicity required relatively long-term exposure.

Since FGF protected against glutamate neurotoxicity, we tested the effects of FGF on the cell death that normally occurs in hippocampal cultures (Mattson and Kater, 1988). When hippocampal pyramidal neurons were cultured in the presence of FGF ( $5 \mathrm{ng} / \mathrm{ml}$ added on culture day 0 and every other day thereafter), neuronal survival was significantly enhanced over a $16 \mathrm{~d}$ period (Fig. 3). This trophic effect of FGF was significant as early as culture day 4 . By culture day 8 , the trophic effect of FGF was striking: whereas only $20 \%$ of the neurons survived until culture day 8 in control cultures, nearly $80 \%$ of the neurons survived in cultures containing FGF. By culture day 16, essen- tially no viable neurons remained in control cultures, whereas over $40 \%$ of the initial number of neurons survived in FGFtreated cultures (Fig. 3). The effects of FGF on neuronal survival were dose dependent; survival in cultures maintained for $16 \mathrm{~d}$ in the presence of $1 \mathrm{ng} / \mathrm{ml}$ FGF was significantly reduced compared with cultures maintained in the presence of $5 \mathrm{ng} / \mathrm{ml} \mathrm{FGF}$ (Fig. 3). A greater FGF concentration $(10 \mathrm{ng} / \mathrm{ml})$ did not further promote neuronal survival (Fig. 3). FGF promotion of survival required the repeated addition of FGF since cultures exposed to FGF only on culture day 0 fared no better than control cultures over a $16 \mathrm{~d}$ period (survival rate was $1 \pm 0.8 \%$ in control cultures, and $3 \pm 1.8$ in FGF-treated cultures; $n=6$ ). The latter finding is consistent with previous reports which indicated that the biological activity of FGF decreases with time in culture (Gospodarowicz and Cheung, 1986; Gospodarowicz et al., 1986; Morrison et al., 1986; Walicke et al., 1986). The survival-promoting effect of FGF was specific in that NGF $(10 \mathrm{ng} / \mathrm{ml})$ did not enhance neuronal survival (Fig. 3). In addition to pyramidal neurons (which comprise greater than $70 \%$ of the neuronal population), the cultures of the present study contain bipolar and stellate neurons (cf. Mattson et al., 1988a). The long-term survival of each of these neuronal types was promoted by FGF since all 3 cell types were absent in control cultures by culture day 16, and all 3 types werc present in FGF-treated cultures.

\section{Opposing effects of FGF and glutamate on neurite outgrowth}

A recent study indicated that FGF promoted neurite outgrowth in cultured hippocampal neurons (Walicke et al., 1986). We confirmed and extended those findings in the present study by examining axonal and dendritic outgrowth in pyramidal-like neurons (Figs. 4, 5; Table 1). FGF enhanced several features of dendritic outgrowth. Numbers of primary dendrites/neuron, total dendrite length/neuron, and branch points/dendrite were each increased significantly by FGF (Figs. 4, 5; Table 1). The increased complexity of dendritic arbors in FGF-treated pyramidal-like neurons was quite striking (Figs. 4,5 ). In the case of axons, total length and number of branch points were increased significantly by FGF. NGF had no effects on these parameters of neurite outgrowth. Since previous work showed that the neurotransmitter glutamate can selectively reduce dendritic outgrowth in hippocampal pyramidal-like neurons (Mattson et al., 1988a), we next examined possible interactive effects of FGF and glutamate.

As expected from our previous studies (Mattson et al., 1988a, b), glutamate ( $50 \mu \mathrm{M})$ caused a significant, 3-fold, reduction in the total length of dendritic arbors (Figs. 4, 5; Table 1). Glutamate also reduced the average number of primary dendrites.

Table 1. Effects of FGF and glutamate on dendritic and axonal outgrowth

\begin{tabular}{lcccc} 
Outgrowth parameter & Control & FGF & Glutamate & FGF + glutamate \\
\hline Total dendrite length $(\mu \mathrm{m})$ & $124 \pm 14$ & $440 \pm 38^{a}$ & $35 \pm 5^{a}$ & $315 \pm 31^{b}$ \\
Primary dendrites & $4.5 \pm 0.5$ & $8.1 \pm 0.7^{a}$ & $2.2 \pm 0.2^{a}$ & $4.4 \pm 0.5$ \\
Branch points/dendrite & $0.1 \pm 0.1$ & $1.1 \pm 0.1^{a}$ & $0.2 \pm 0.1$ & $1.1 \pm 0.3^{b}$ \\
Total axon length $(\mu \mathrm{m})$ & $248 \pm 17$ & $437 \pm 36^{a}$ & $315 \pm 16$ & $406 \pm 33^{b}$ \\
Branch points/axon & $3.1 \pm 0.6$ & $8.7 \pm 1.0^{a}$ & $4.0 \pm 0.4$ & $4.8 \pm 0.7$
\end{tabular}

Values represent the mean \pm SEM of determinations made on 11-14 pyramidal-like neurons on culture day 5 . FGF was added on days 1,2 , and 4; glutamate was added on day 2 .

${ }^{a} p<0.01-0.001$ vs control.

${ }^{b} p<0.01-0.001$ vs control or glutamate. 

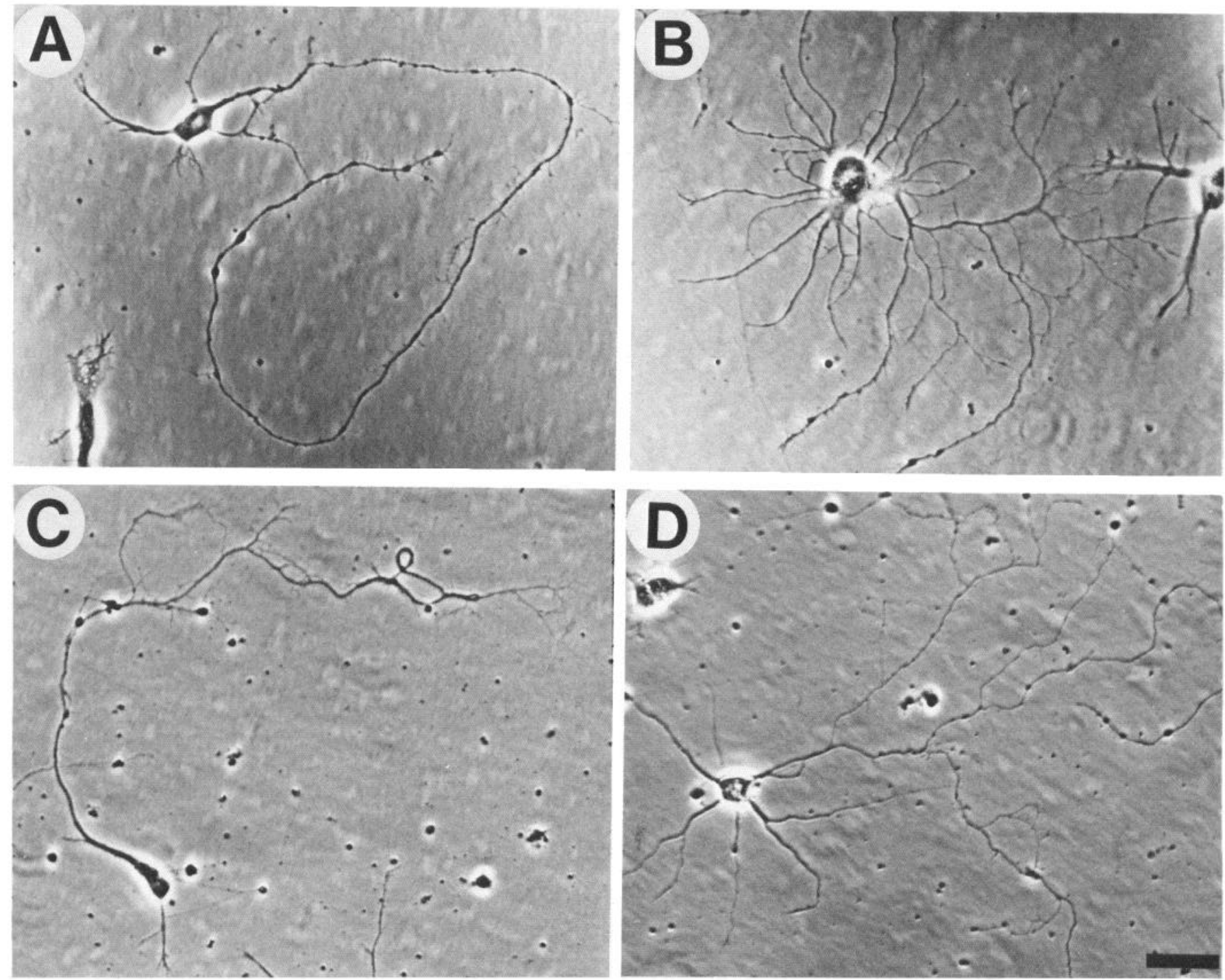

Figure 4. Interactive effects of FGF and glutamate on neurite outgrowth in pyramidal neurons. Representative micrographs of pyramidal neurons (culture day 5) cultured under the following conditions: $A$, Control conditions in the absence of FGF or glutamate. $B$, In the presence of FGF ( $5 \mathrm{ng} / \mathrm{ml}$ final concentration, added on culture days, 0,2 , and 4). $C$, In the presence of glutamate (50 $\mu \mathrm{M}$ added on culture day 2$)$. $D$, In the presence of FGF ( $5 \mathrm{ng} / \mathrm{ml}$ final concentration, added on culture days 0,2 , and 4$)$ and glutamate (50 $\mu \mathrm{M}$ added on culture day 2$)$. Scale bar, $25 \mu \mathrm{m}$.

Axonal length and numbers of branch points were not affected significantly by glutamate (Table 1 ). The important new finding of the present study was that the negative effects of glutamate on dendritic outgrowth were completely prevented by FGF. In fact, FGF enhanced outgrowth even in the presence of $50 \mu \mathrm{M}$ glutamate (Figs. 4, 5; Table 1). Total dendrite length in neurons exposed to both FGF and glutamate was 9 times that in neurons cultured in the presence of glutamate alone (Table 1). Numbers of branch points/dendrite were also significantly greater in neurons exposed to both FGF and glutamate than in cultures exposed to glutamate alone. The average number of primary dendrites in neurons exposed to both FGF and glutamate was intermediate to the numbers of primary dendrites/neuron in cultures exposed to FGF or glutamate alone (Table 1). The total length of pyramidal neuron axonal arbors was similar in cultures treated with FGF or FGF plus glutamate. NGF $(10 \mathrm{ng} / \mathrm{ml})$ did not prevent the reductions in dendritic outgrowth caused by glutamate (not shown). Taken together, these data indicate that FGF enhances neurite outgrowth and branching and thereby increases the complexity of neuritic arbors; FGF can antagonize glutamate-induced inhibition of dendrite outgrowth.

\section{Mechanisms of action of FGF and glutamate}

The trophic actions of neuronal growth factors are believed to be mediated, at least in part, by changes in gene and/or protein expression (Greene and Shooter, 1980), which generally require periods of hours to days to be accomplished. On the other hand, glutamate neurotoxicity is apparently mediated by large, rapid (sec to $\mathrm{min}$ ) rises in intracellular calcium resulting from influx through plasma membrane channels (Choi, 1988; Mattson et al., 1988a, d, 1989). In the present study, we made an initial characterization of the mechanism by which FGF reduces glutamate neurotoxicity. 

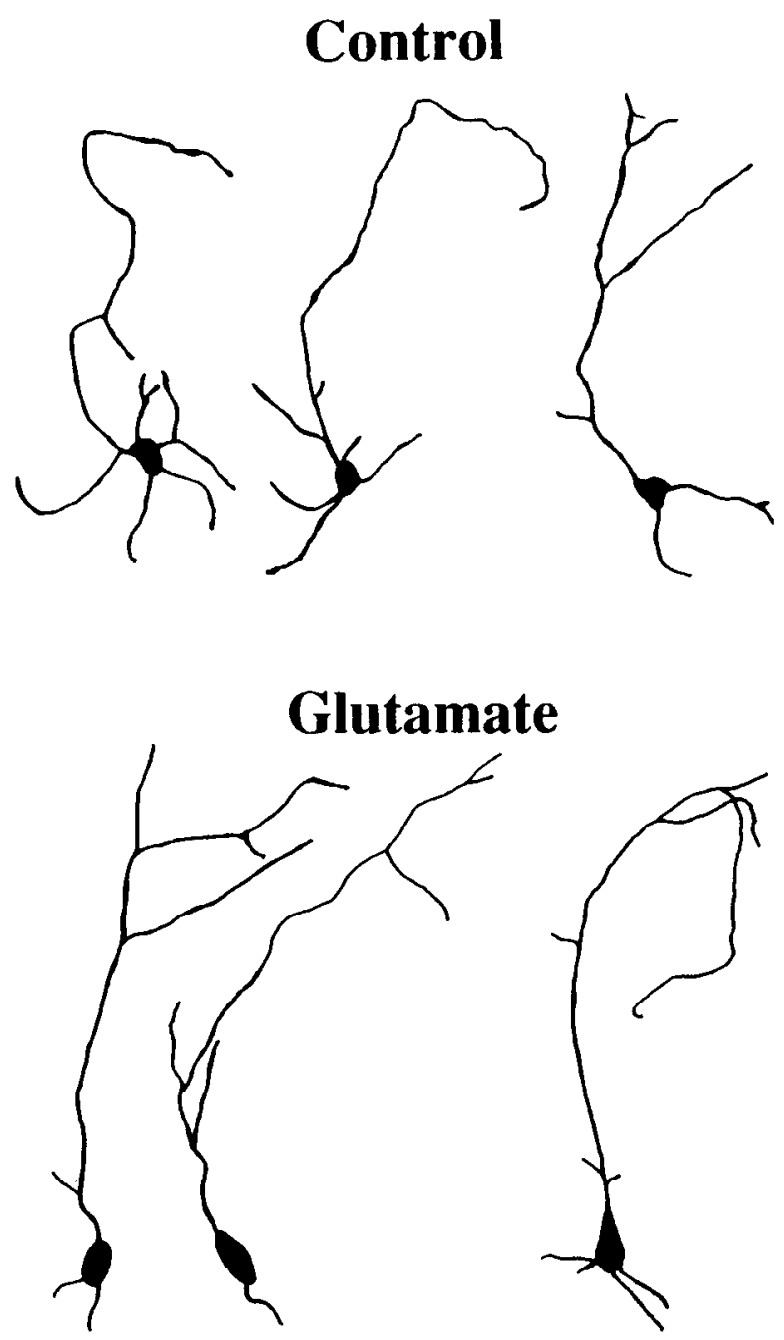

\section{FGF}
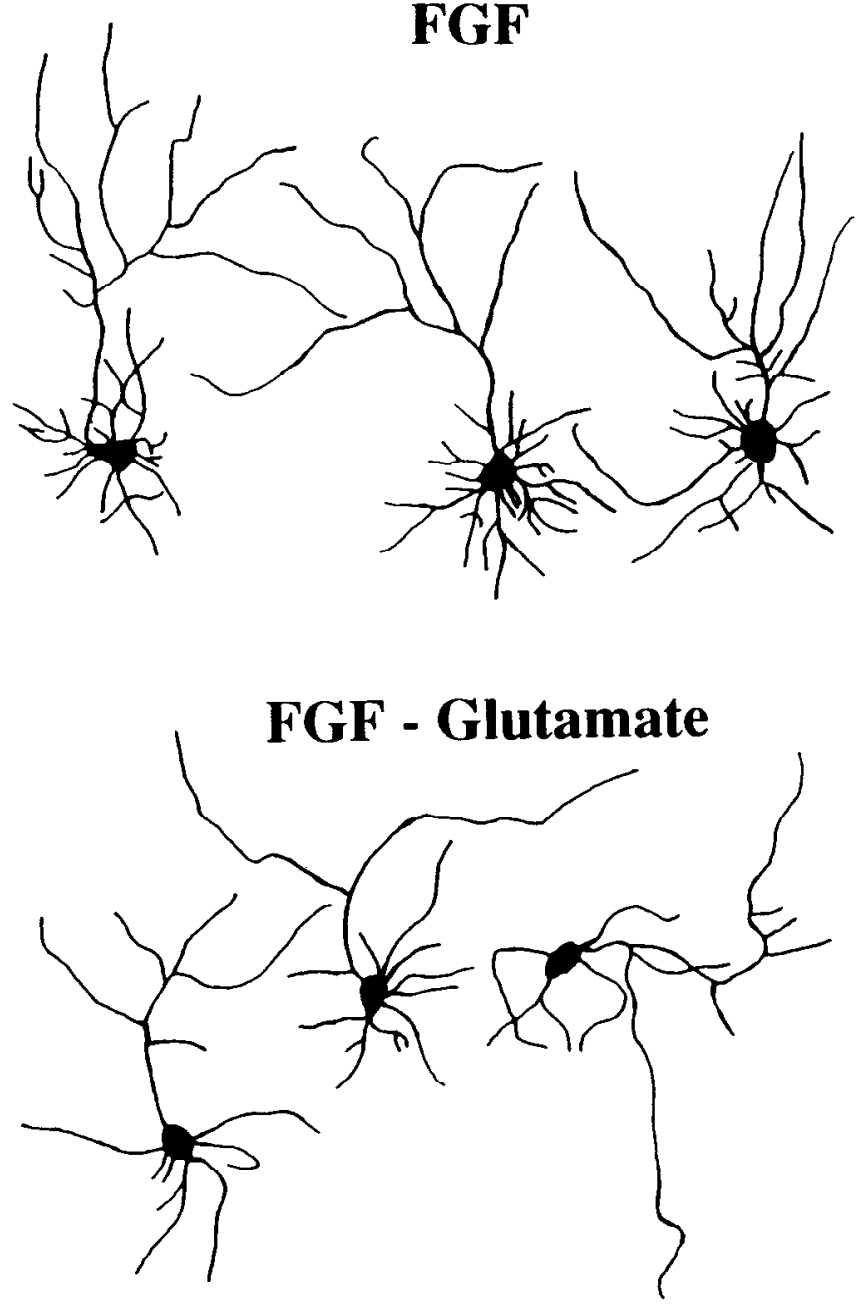

Figure 5. Effects of FGF and glutamate on pyramidal neuron cytoarchitecture. Tracings of pyramidal neurons ( $5 \mathrm{~d}$ in culture) cultured under the indicated conditions. FGF ( $5 \mathrm{ng} / \mathrm{ml}$ final concentration, added on culture days 0,2 , and 4); glutamate (50 $\mu \mathrm{M}$ added on culture day 2 ). These micrographs and tracings are representative of $45-60$ pyramidal neurons examined.

\section{Involvement of protein synthesis}

Our finding that the protective effect of FGF against glutamate neurotoxicity required pretreatment with FGF for several hours prior to addition of glutamate was consistent with the possibility that mRNA and/or protein synthesis was required for FGF's actions. We therefore tested whether inhibitors of mRNA (actinomycin D) or protein (cycloheximide) syntheses modified the neuroprotective effects of FGF. On culture day 4, neurons in control or FGF-treated cultures were exposed to either $5 \mu \mathrm{M}$ actinomycin D or $1 \mu \mathrm{M}$ cycloheximide for $18 \mathrm{hr}$, and then the cultures were exposed to glutamate ( 200 or $500 \mu \mathrm{M}$ ) for $24 \mathrm{hr}$. Actinomycin D and cycloheximide alone had no significant effect on cell survival over this time period (Table 2). As expcctcd, glutamate reduced pyramidal neuron survival and FGF antagonized glutamate neurotoxicity. In the absence of FGF, actinomycin $D$ and cycloheximide each enhanced the neurotoxicity of $200 \mu \mathrm{M}$ glutamate, suggesting that ongoing transcription and translation may normally afford protection against glutamate neurotoxicity. Actinomycin D and cycloheximide each significantly negated the protective effects of FGF against glutamate neurotoxicity. When FGF-treated cultures were exposed to ac- tinomycin D or cycloheximide prior to addition of glutamate, there was a significant reduction in neuronal survival to levels similar to those seen in cultures lacking FGF (Table 2). These results indicated that mRNA and protein synthesis are each required in order for FGF to protect against glutamate neurotoxicity.

\section{Calcium}

Since our previous studies demonstrated that calcium influx is both necessary and sufficient to account for glutamate-induced neurodegeneration in hippocampal neurons (Mattson et al., 1988a, d, 1989; Mattson and Kater, 1989a), it was of interest to examine intracellular calcium levels in neurons exposed to FGF and glutamate. Basal levels of intracellular calcium were similar in pyramidal neurons in control cultures $(72 \pm 2.9 \mathrm{nM}$; $n=12)$ and in cultures maintained in the presence of FGF ( 66 $\pm 1.9 \mathrm{nM} ; n=13$ ). Under these basal conditions, intracellular calcium levels were below $100 \mathrm{~nm}$ in all 63 neurons examined. In control cultures, maintained in the absence of FGF, glutamate $(500 \mu \mathrm{M})$ caused intracellular calcium levels to rise well over $100 \mathrm{~nm}$ in the majority of pyramidal neurons within $30 \mathrm{~min}$ of exposure (Fig. 6). Calcium responses (levels elevated over 100 

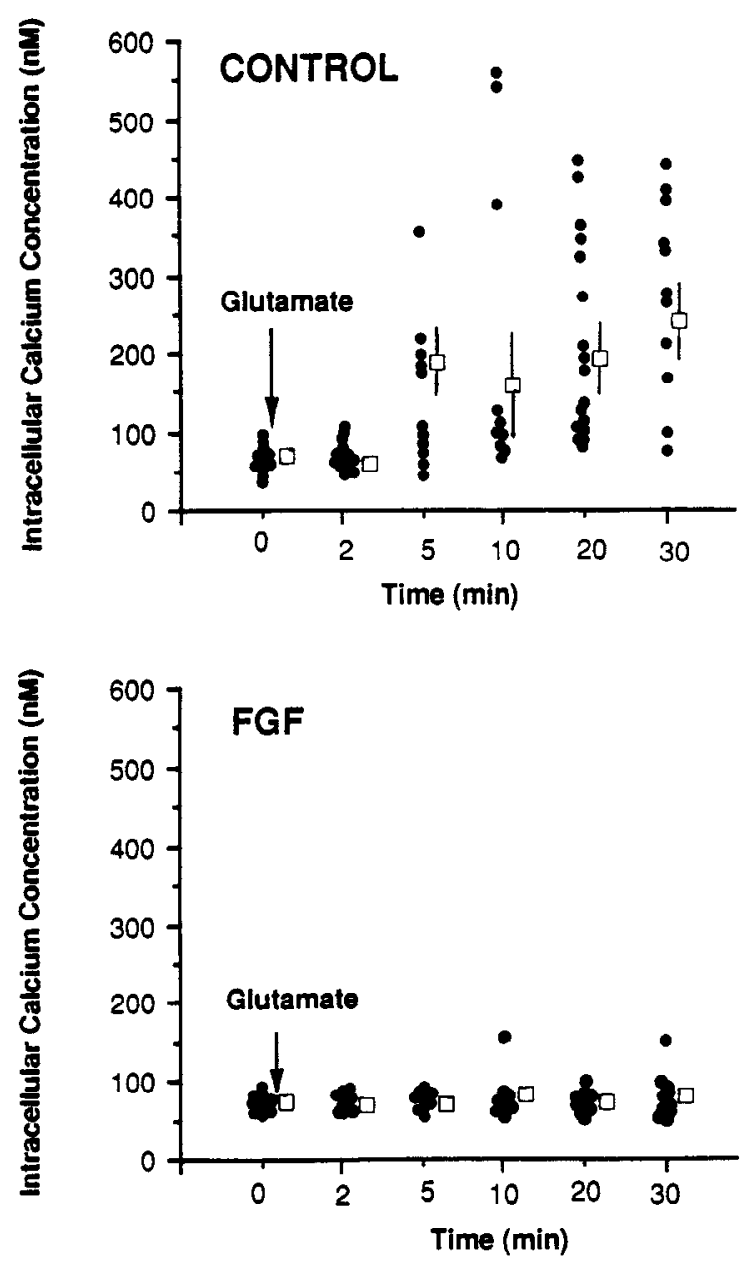

Figure 6. FGF reduces the glutamate-induced rises in intracellular calcium levels in hippocampal neurons. Intracellular calcium levels in 5 -d-old pyramidal-like neurons were quantified using fura-2 methods. Top, Control (no FGF); bottom $(5 \mathrm{ng} / \mathrm{ml}$ final concentration, added on culture days 0,2 , and 4 , and included in the medium for fura- 2 analysis), FGF. Closed circles represent values for individual neurons; open squares and error bars are means \pm SEM of the individual values $(n=10-18$; where error bars are not seen, the squares obscure the SEM).
Table 2. Effects of actinomycin D and cycloheximide on glutamateinduced neurodegeneration in control and FGF-treated cultures

\begin{tabular}{ll} 
& $\begin{array}{l}\text { Pyramidal neuron } \\
\text { survival } \\
\text { (\% of initial number) }\end{array}$ \\
Treatment & $93 \pm 3.0$ \\
\hline Control & $97 \pm 1.9$ \\
FGF $(5 \mathrm{ng} / \mathrm{ml}$, days 0 and 4) & $96 \pm 1.8$ \\
Actinomycin D (ActD, 5 $\mu \mathrm{M})$ & $92 \pm 3.9$ \\
Cycloheximide (Cyclo, $1 \mu \mathrm{M})$ & $96 \pm 1.8$ \\
FGF + ActD & $95 \pm 2.1$ \\
FGF + Cyclo & $40 \pm 5.3^{a}$ \\
Glutamate $(200 \mu \mathrm{M})$ & $24 \pm 3.5^{a}$ \\
Glutamate $(500 \mu \mathrm{M})$ & $88 \pm 3.8^{b}$ \\
FGF + glutamate $(200 \mu \mathrm{M})$ & $49 \pm 4.9^{b}$ \\
FGF + glutamate $(500 \mu \mathrm{M})$ & $27 \pm 3.3^{a, b}$ \\
ActD + glutamate $(200 \mu \mathrm{M})$ & $16 \pm 3.2^{a}$ \\
ActD + glutamate $(500 \mu \mathrm{M})$ & $23 \pm 3.4^{a . b}$ \\
Cyclo + glutamate $(200 \mu \mathrm{M})$ & $22 \pm 4.0^{a}$ \\
Cyclo + glutamate $(500 \mu \mathrm{M})$ & $23 \pm 2.7^{c}$ \\
FGF + ActD + glutamate $(200 \mu \mathrm{M})$ & $21 \pm 5.0^{c}$ \\
FGF + ActD + glutamate $(500 \mu \mathrm{M})$ & $24 \pm 4.0^{c}$ \\
FGF + Cyclo + glutamate $(200 \mu \mathrm{M})$ & $23 \pm 4.3^{c}$ \\
FGF + Cyclo + glutamate $(500 \mu \mathrm{M})$ &
\end{tabular}

Values represent means \pm SEM of determinations made on 8 fields in 2 separate dishes. FGF $(5 \mathrm{ng} / \mathrm{ml})$ was added on culture days 0,2 , and 4 . Actinomycin $D$ and cycloheximide were added $18 \mathrm{hr}$ prior to glutamate treatment which was added on culture day 5 ; cell survival was assessed $24 \mathrm{hr}$ later.

" $p<0.001$ compared with control.

" $p<0.01-0.001$ compared with values for cultures treated with corresponding levels of glutamate alone.

$c p<0.01$ compared with cultures treated with FGF + glutamate.

nM) were first seen $5 \mathrm{~min}$ posttreatment, and by $30 \mathrm{~min}$ posttreatment intracellular calcium levels were elevated 2- to 8-fold in $70-85 \%$ of the pyramidal neurons examined. In some neurons, intracellular calcium levels were not elevated following exposure to glutamate (Fig. 6). Since approximately $20-30 \%$ of the pyramidal neurons were resistant to glutamate neurotoxicity (cf. Fig. 1) and because previous data indicated that calcium

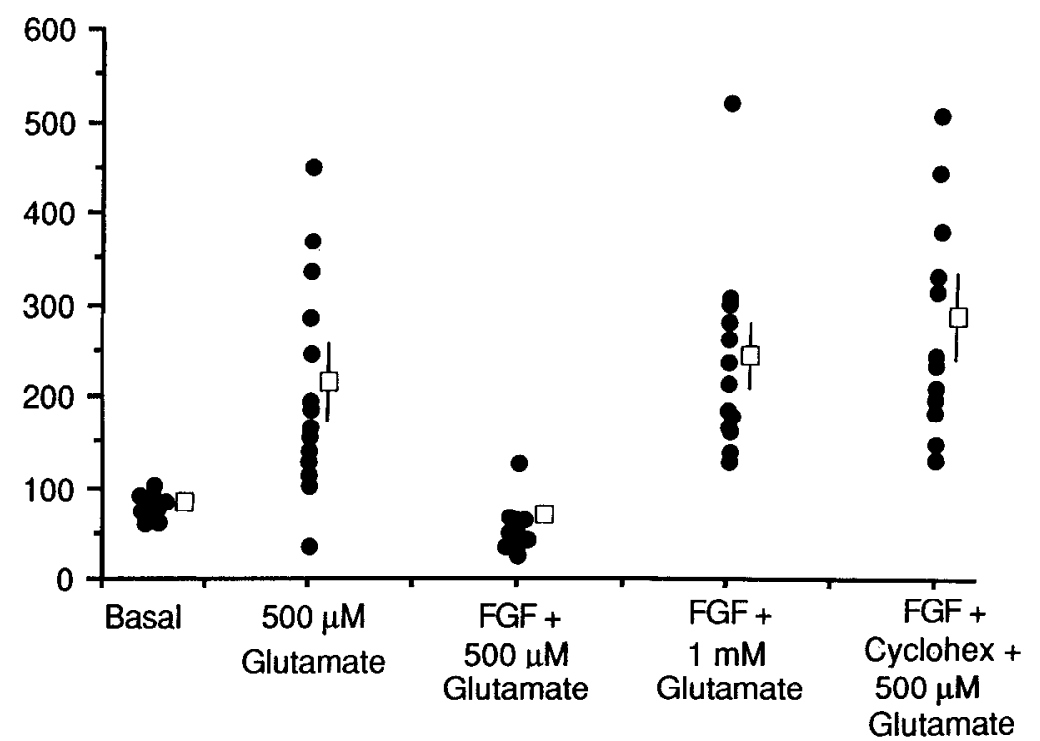

Figure 7. Effects of FGF and cycloheximide on glutamate-induced rises in intracellular calcium levels. Intracellular calcium levels in 5-d-old pyramidallike neurons were determined prior to and $30 \mathrm{~min}$ following exposure to the indicated treatments. Closed circles represent values for individual neurons; open squares and error bars are the mean \pm SEM of the individual valucs (whcre error bars are not seen, the squares obscure the SEM). The basal value represents the cumulative mean \pm SEM of intracellular calcium levels in pyramidal neurons prior to experimental treatment $(n=68)$. All other values represent the posttreatment levels of calcium $(n=12-18)$. 


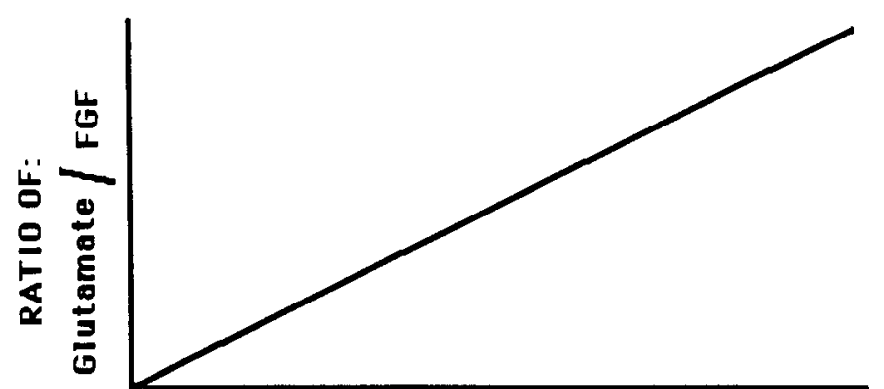

Intracellular Calcium Concentration
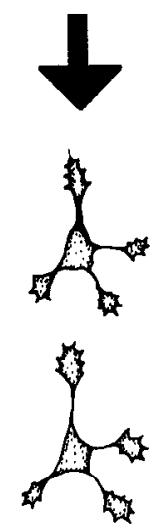

MEUR I TE DUTEROUTH
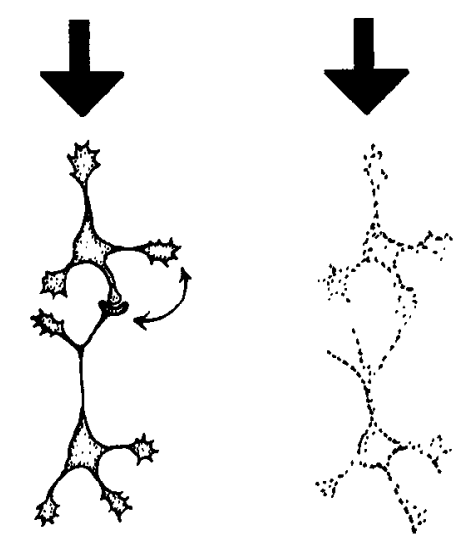

\section{PLAST I CITY DEGENERATION}

Figure 8. Model for interactions of glutamate and FGF in the development, plasticity, and degeneration of hippocampal neuroarchitecture. FGF has positive effects, and glutamate has negative effects on neurite outgrowth and cell survival. When the ratio of glutamate/FGF input to a neuron is low, as is likely to occur during early hippocampal development, neurite outgrowth is promoted. As the glutamate/FGF ratio increases, circuitry forms. In the mature nervous system changes in levels of FGF and glutamate input may be involved in adaptive changes in neuroarchitecture (plasticity). When the glutamate/FGF ratio becomes excessively high, neurodegeneration occurs. Intracellular calcium appears to play an important role as a mediator of glutamate and FGF actions. Glutamate increases intracellular calcium levels, which results in the inhibition of outgrowth or neurodegeneration. FGF tends to keep intracellular calcium levels low, thereby promoting neurite outgrowth and cell survival. See text for further details.

responses are not elicited by glutamate in resistant neurons (Mattson et al., 1989), it seemed likely that the neurons which showed no calcium response were also resistant to glutamate neurotoxicity. This possibility was confirmed in one experiment in which fura- 2 measurements of calcium levels were followed by examination of cell survival $24 \mathrm{hr}$ later. In all cases, the cells which showed calcium responses to glutamate $(n=11)$ subsequently degenerated, while those in which calcium did not change appreciably $(n=4)$ survived.

Since FGF raised the threshold for glutamate neurotoxicity and dendritic pruning, it was of interest to determine whether FGF modified the calcium response to glutamate. Accordingly, cultures maintained in the presence of FGF were exposed to $500 \mu \mathrm{M}$ glutamate and intracellular calcium levels were monitored with fura-2. There was a striking reduction in the percentage of cells showing calcium responses to glutamate in cultures maintained in the presence of FGF (Fig. 6). Indeed, intracellular calcium levels were elevated above $100 \mathrm{~nm}$ in only 2 of the 44 neurons examined between 5 and 30 min posttreatment. In order to further support a relationship between rises in intracellular calcium and cell death, we exposed cultures to a level of glutamate (1 $\mathrm{mm}$ ) which caused neurodegeneration even in the presence of FGF (cf. Fig. 1). Under these conditions, 12 of 13 pyramidal neurons examined had elevated levels of intracellular calcium after 30 min of exposure (Fig. 7) and subsequently died. In a final experiment, we tested the effects of cycloheximide on calcium responses to glutamate in FGF-treated cells. When FGF-containing cultures wcrc pretreated with 1 $\mu \mathrm{M}$ cycloheximide for $18 \mathrm{hr}$ and then exposed to $500 \mu \mathrm{M}$ glutamate, all 12 neurons had intracellular calcium levels which were greater than $100 \mathrm{~nm}$ (Fig. 7). Taken together, these data indicate that FGF antagonizes glutamate-induced rises in intracellular calcium by a mechanism requiring protein synthesis.

\section{Discussion}

The present study provides support for the hypothesis that growth factors and neurotransmitters can interact in the development and degeneration of neuroarchitecture (Fig. 8). Acting separately, FGF promoted, while glutamate reduced, neurite outgrowth and cell survival. Combined exposure to FGF and glutamate resulted in intermediate levels of neurite outgrowth and cell survival. These findings suggest that the relative levels of growth factors and neurotransmitters to which a neuron is exposed can determine whether a particular neuron survives or dies and whether its neuritic arbors expand or regress. Clearly, these kinds of neuroarchitectural correlates of growth factor and neurotransmitter actions would have profound effects on the establishment, plasticity, and degeneration of neural circuitry.

FGF and glutamate interactions in hippocampal development

\section{FGF alone}

The results of the present and previous studies suggest that FGF may be an endogenous neurotrophic and neuritotropic signal in the developing hippocampus. FGF is present at high levels in the developing brain (Gospodarowicz et al., 1986), CNS glia and neurons are known to produce FGF (Caday et al., 1988), hippocampal neurons bear FGF receptors (Walicke et al., 1989), and the survival and outgrowth of cultured neurons from several brain regions is promoted by FGF (Morrison et al., 1986; Walicke, 1988). In addition, glial celis (Banker, 1980) and a putative autocrine factor(s) (Mattson and Kater, 1988) provide trophic and neuritotropic support for cultured hippocampal neurons, and astroglial-derived FGF has been shown to promote neurite outgrowth in other CNS neurons (Hatten et al., 1988). Thus, FGF may serve as a developmental signal in many areas of the nervous system. In the present study, FGF incrcased the numbers of primary dendrites and the total dendrite length and branching, as well as axon length and branching. These effects of FGF on neurite outgrowth resulted in an increased complexity of pyramidal neuron dendritic and axonal arbors. The information-coding capabilities of nervous systems are determined in large part by the presence of the component neurons and their specific neuritic shapes and synaptic contacts. IfFGF has similar trophic and neuritotropic effects in vivo, then one role for FGF would be to increase the integrative capabilities of hippocampal circuitry.

\section{Glutamate alone}

In contrast to FGF, glutamate reduced neuronal survival and dendritic outgrowth. Such actions of glutamate are believed to play important roles during hippocampal development (Matt- 
son, 1988; Mattson et al., 1988a, b). Axonal growth cones are known to release neurotransmitters (Hume et al., 1983; Young and Poo, 1983; Mattson et al., 1988b), and our recent findings indicate that endogenous glutamate, released from entorhinal axons, selectively inhibits dendrite outgrowth in target hippocampal neurons (Mattson et al., 1988b). Thus, the developmental role of glutamate appears to be opposite that of FGF, at least with regard to the elaboration of dendritic architecture.

\section{Combined actions of FGF and glutamate}

FGF reduced the negative effects of glutamate on pyramidal neuron survival and dendritic outgrowth. These findings suggest that these 2 different types of signals might interact to "adjust" neuronal numbers and to "sculpt" neuritic architecture during brain development. Previously, such sculpting roles for growth factors and neurotransmitters have been studied separately. For example, data suggest that NGF or other growth factors may play roles in the development of the vertebrate PNS where there is an initial overproduction of sensory and motor neurons followed by a regression in neuronal numbers as synaptic connections are established (Bennett, 1983; Oppenheim, 1987). Individual trophic substances may also play important roles in adaptive adjustments in neuroarchitecture that occur in development and adult plasticity (Purves, 1986). On the other hand, many different neurotransmitters, acting alone, can inhibit neurite outgrowth (see Mattson, 1988; Lipton and Kater, 1989, for reviews). Neurotransmitters such as glutamate can also induce neuronal death (Choi, 1988; Mattson, 1988).

Since individual neurons in vivo are likely to be exposed to both growth factors and neurotransmitters, it would be expected that relative levels of growth factors and ncurotransmitters determine whether dendrites grow, stop, or regress, as well as whether neurons survive. For example, transient expression of FGF may promote the initial proliferation of dendritic arbors even in the presence of glutamate. When FGF levels fall, glutamate is effective in inhibiting dendritic outgrowth and circuitry becomes consolidated. Specific localized outgrowth responses would be determined by the pairing of specific growth factors and neurotransmitters with their corresponding receptors. Individual dendrites might be selectively pruned by glutamate (Mattson et al., 1988a), or a growth factor released locally might enhance the outgrowth of one axonal growth cone (Gundersen and Barrett, 1980). These are the kinds of localized and specific intercellular signaling processes which might be expected to underlie the development of a cellular ensemble as structurally complex as the nervous system.

\section{Intracellular mechanisms of FGF and glutamate actions}

We were able to gain insight into the mechanisms by which FGF promotes survival by studying interactions between FGF and glutamate. The ability of FGF to reduce glutamate neurotoxicity required RNA and protein syntheses since FGF did not reduce glutamate neurotoxicity in cultures pretreated with actinomycin D or cycloheximide. Interestingly, our results also indicate that ongoing transcription and translation may be important for protection against glutamate neurotoxicity in the absence of FGF. Thus, we found that actinomycin $\mathrm{D}$ and cycloheximide each significantly enhanced glutamate neurotoxicity in cultures maintained in the absence of FGF. By analogy to NGF, the trophic influence of FGF may require uptake and retrograde transport to the soma, where gene expression and protein synthesis may be influenced (Walicke et al., 1989). On the other hand, recent work by Martin et al. (1988) indicates that protein synthesis inhibitors reduce the cell death that normally occurs in NGF-deprived peripheral neurons. We found that extended exposure (several days) of hippocampal neurons to cycloheximide or actinomycin D reduced cell survival both in the presence and absence of FGF. Therefore, FGF's mechanism of trophic action appears to be somewhat different from that of NGF. It seems likely that different types of growth factors may act differently to promote neuronal survival. Given the precedence for the regulation of gene expression by different growth factors (e.g., Leonard et al., 1987), it will certainly be of interest to identify proteins and encoding genes whose expression in hippocampal neurons is affected by FGF.

Recent investigations have begun to reveal the cellular mechanisms by which glutamate influences cell survival and dendritic outgrowth. Data from studies of hippocampal (Mattson et al., 1988a, d, 1989) and cortical (Choi, 1987, 1988) cell cultures indicate that glutamate-induced calcium influx through plasma membrane channels plays a causal role in neurodegeneration. Thus, glutamate causes large rises in intracellular calcium levels (Connor et al., 1988; Mattson et al., 1988d, 1989), blockade of calcium channels or removal of extracellular calcium prevents glutamate neurotoxicity (Choi, 1987; Mattson et al., 1988a, 1989), and calcium ionophore A23187 is neurotoxic (Mattson et al., 1988a). The large rises in intracellular calcium induced by glutamate may activate calcium-dependent proteases and cause the generation of free radicals, which, in turn, lead to the breakdown of the cytoskeleton and membranes (Dykens et al., 1987; Siesjo, 1988; Siman and Noszek, 1988). The outgrowthregulating actions of subtoxic levels of glutamate also involve intraccllular calcium. Glutamate and related cxcitatory amino acids cause sustained gradients of intracellular calcium with high levels in the dendrites and lower levels in the soma and axon (Connor et al., 1988; M. P. Mattson, unpublished observations). Data from pharmacological studies in cultured hippocampal pyramidal neurons indicate that calcium influx localized to the dendrites is responsible for the selective inhibitory effects of glutamate on dendrite outgrowth (Mattson et al., 1988a, d). Presumably, the localization of glutamate receptors to dendrites is the basis for the local rises in intracellular calcium and the resulting effects on dendrite outgrowth.

Our data indicate that intracellular calcium is a common regulatory locus for both the neurodegenerative actions of glutamate and the neuroprotective actions of FGF. FGF alone did not significantly alter basal levels of intracellular calcium. However, FGF prevented rises in intracellular calcium normally caused by glutamate, and also reduced glutamate neurotoxicity. In effect, FGF raised the threshold for the large, sustained calcium responses and subsequent neurodegeneration normally caused by glutamate. Finally, cycloheximide prevented FGF from protecting against both glutamate-induced rises in intracellular calcium levels and neurotoxicity. Taken together, these data are consistent with the possibility that FGF acts on some component of the cellular systems for calcium homeostasis. For example, it is conceivable that FGF increases the numbers or efficiency of calcium extrusion systems such as the plasma membrane ATPase or the $\mathrm{Na}^{+} / \mathrm{Ca}^{2+}$ exchanger (Blaustein, 1988). Alternatively, FGF might modify calcium channel numbers or function. There is, in fact, evidence that growth factors influence calcium-regulating systems in other cell types. For example, NGF may influence the expression of calcium channels in PC12 cells (Streit and Lux, 1987; Lewis et al., 1988). An additional 
potential site of modification by FGF is the glutamate receptor. A reduction in numbers of glutamate receptors or a blockage of receptor function could account for the observed effects of FGF on intracellular calcium levels and glutamate neurotoxicity.

Finally, it should be pointed out that although the fura- 2 data presented here provide important information on overall changes in intracellular calcium levels with time, they do not provide a comprehensive picture of underlying changes between subcellular compartments. For example, previous studies of responses of endocrine cells to hormones and neural cells to neurotransmitters suggest that these primary signals can induce the "cycling" of calcium between cell compartments or across the plasma membrane (Alkon and Kasmussen, 1988). Furthermore, different intracellular messenger systems such as the inositol phospholipid system may interact with the calcium system in a spatially and/or temporally specific manner (Alkon and Rasmussen, 1988; Mattson et al., 1988d). It is therefore possible that glutamate or FGF activate cycling mechanisms or multiple messenger pathways not considered in the present study. Clearly, it will be important to examine the actions and interactions of growth factors and neurotransmitters on cellular calciumregulating systems in future studies.

\section{Implications for abnormal neurodegeneration}

The hippocampus is an important site of neuropathology. Pyramidal neurons of regions $\mathrm{CA} 1$ and $\mathrm{CA} 3$ are selectively vulnerable in several disorders, including Alzheimer's disease (VanHoesen et al., 1986; Maragos et al., 1987), epilepsy (Sloviter, 1983), and stroke (Rothman and Olney, 1986). Results of studies done both in situ (Sloviter, 1983; Simon et al., 1984; McDonald et al., 1987) and in vitro (Mattson et al., 1988a; Mattson and Kater, 1989b) are consistent with glutamate involvement in this selective neuronal vulnerability. Cell culture studies of mitotic sister pyramidal neurons (Mattson et al., 1989) and pyramidal neurons isolated from different hippocampal regions (Mattson and Kater, 1989b) provide evidence that vulnerability to glutamate neurotoxicity results from intrinsic differences in pyramidal neurons acquired during development. The cellular basis of the differences is not clear but may result from differences in the expression of glutamate receptors (Mattson et al., 1988a, d; Mattson and Kater, 1988, 1989b), calciumbinding proteins (Hoffmann et al., 1988), or metabolic pathways (Koh and Choi, 1988). A second major hypothesis for age- and disease-associated neurodegeneration postulates that deficiencies in specific growth factors lead to the loss of neurons (Appel, 1986; Fine and Rubin, 1988). For example, among the data supporting this hypothesis for Alzheimer's disease are basal forebrain cholinergic neurons are lost in Alzheimer's disease; NGF is trophic for basal forebrain cholinergic neurons (Hefti et al., 1984; Shelton and Reichardt, 1986); NGF is present at high levels in the hippocampus; hippocampus is a target for basal forebrain neurons (Korsching et al., 1985; Korsching, 1986). FGF's trophic and neuritotropic actions on hippocampal neurons are consistent with a role for this growth factor in abnormal hippocampal neurodegeneration.

Our data provide a basis for the merger of the 2 hypotheses for abnormal neurodegeneration just described. We propose that imbalances in relative levels of growth factors and neurotransmitters can lead to neurodegeneration (Fig. 8). Such imbalances could arise by large reductions in FGF levels, large rises in glutamate, or combined moderate reductions in FGF and elevations in glutamate. This model is consistent with the fact that
FGF was able to reduce neurodegeneration normally seen in response to moderate (100-500 $\mu \mathrm{M}$ ) levels of glutamate but did not prevent cell death in cultures exposed to high (millimolar) levels of glutamate. While the present findings provide the first direct evidence that growth factors can reduce excitatory amino acid neurotoxicity, previous observations in situ are consistent with an interaction between trophic factors and excitatory amino acids. For example, Tulipan et al. (1988) found that grafts of embryonic striatum into the striatum of adult rats protect against kainic and quinolinic acid neurotoxicity. In vitro studies suggest that a trophic substance released by the embryonic striatal tissue is responsible for protection against excitotoxicity (Whetsell et al., 1989). Further work should provide important information on the extent to which growth factors can modify excitatory amino acid-induced neurodegeneration in vivo.

In the intact hippocampus, further complexity is added when one considers the involvement of additional neurotransmitters and growth factors. For example, GABA provides the major inhibitory input to pyramidal neurons in situ, and activation of GABA receptors prevents glutamate-induced degeneration in cultured pyramidal neurons (Mattson and Kater, 1989a). These data suggest that reductions in GABAergic systems might lead to neurodegeneration. Indeed, recent studies done in situ indicate that GABA agonists can reduce transneuronal death in the substania nigra caused by excitatory amino acid injection into the caudate (Saji and Reis, 1987). Given our sparse knowledge of growth factor actions in the CNS, it is also reasonable to consider that growth factors other than FGF play roles in the maintenance of hippocampal neurons. The demonstration of interactive effects of neurotransmitters and growth factors on individual neurons suggests that neurodegeneration in aging or specific diseases may result from alterations in single or multiple signaling systems. Finally, the importance of intracellular calcium in the generative and degenerative actions of FGF and glutamate suggest that abnormalities in cellular calcium-regulating systems might also lead to untimely neurodegeneration (see Gibson and Peterson, 1987). It may therefore prove useful to target signal transduction systems for growth factors and neurotransmitters in therapeutic and preventative approaches to neurodegenerative disorders.

\section{References}

Alkon, D. L., and H. Rasmussen (1988) A spatial-temporal model of ccll activation. Science 239: 998-1004.

Appel, S. H. (1986) A unifying hypothesis for the cause of amyotrophic lateral sclerosis, Parkinsonism, and Alzheimer disease. Ann. Neurol. 10: 499-505.

Balazs, R., N. Hack, and O. S. Jorgensen (1988) Stimulation of the $\mathrm{N}$-methyl-D-aspartate receptor has a trophic effect on differentiating cerebellar granule cells. Neurosci. Lett. 87: 80-86.

Banker, G. A. (1980) Trophic interactions between astroglial cells and hippocampal neurons in culture. Science 209: 809-810.

Bennett, M. R. (1983) Development of neuromuscular synapses. Physiol. Rev. 63: 915-1048.

Black, I. B. (1986) Trophic molecules and the evolution of the nervous system. Proc. Natl. Acad. Sci. USA. 83: 8249-8252.

Blaustein, M. P. (1988) Calcium transport and buffering in neurons. Trends Neurosci. 11: 438-443.

Bostwick, J. R., S. H. Appel, and J. R. Perez-Polo (1987) Distinct influences of nerve growth factor and a central cholinergic trophic factor on medial septal explants. Brain Res. 422: 92-98.

Caday, C. G., A. Mirzabegian, J. Prosser, M. Klagsbrun, and S. P. Finklestein (1988) Fibroblast growth factor levels in developing brain. Soc. Neurosci. Abstr. 14: 363 .

Chang, F. L. F., and W. T. Greenough (1983) Transient and enduring 
morphological correlates of synaptic activity and efficacy in the rat hippocampal slice. Brain Res. 309: 35-46.

Choi, D. W. (1987) Ionic dependence of glutamate neurotoxicity. J. Neurosci. 7: 369-379.

Choi, D. W. (1988) Glutamate neurotoxicity and diseases of the nervous system. Neuron 1: 623-634.

Cline, H. T., E. A. Debski, and M. Constantine-Paton (1987) N-methyl-D-aspartate receptor antagonist desegregates eye-specific stripes. Proc. Natl. Acad. Sci. USA 84: 4342-4345.

Cohan, C. S., J. A. Connor, and S. B. Kater (1987) Electrically and chemically mediated increases in intracellular calcium in neuronal growth cones. J. Neurosci. 7: 3588-3599.

Connor, J. A., W. J. Wadman, P. E. Hockberger, and R. K. S. Wong (1988) Sustained dendritic gradients of $\mathrm{Ca}^{2+}$ induced by excitatory amino acids in CA1 hippocampal neurons. Science 240: 649-653.

Cowan, W. M., J. W. Fawcett, D. D. M. O'Leary, and B. B. Stanfield (1984) Regressive events in neurogenesis. Science 225: 1258-1265.

Coyle, J. T., and R. Schwarcz (1976) Lesion of striatal neurons with kainic acid provides a model for Huntington's Chorea. Nature 263: 244-246.

Crutcher, K. A. (1987) Sympathetic sprouting in the central nervous system: A model for studies of axonal growth in the mature mammalian brain. Brain Res. Rev. 12: 203-233.

Desmond, N., and W. B. Levy (1983) Synaptic correlates of associative potentiation/depression: An ultrastructural study in the hippocampus. Brain Res. 265: 21-31.

Dotti, C. G., C. A. Sullivan, and G. A. Banker (1988) The establishment of polarity by hippocampal neurons in culture. J. Neurosci. 8 : $1454-1468$.

Dykens, J. A., A. Stern, and E. Trenkner (1987) Mechanism of kainate toxicity to cerebellar neurons in vitro is analogous to reperfusion tissue injury. J. Neurochem. 49: 1222-1228.

Fine, R. E., and J. B. Rubin (1988) Specific trophic factor-receptor interactions: Key selective elements in brain development and "regeneration." J. Am. Geriatric Soc. 36: 457-466.

Gibson, G. E., and C. Peterson (1987) Calcium and the aging nervous system. Neurobiol. Aging 8: 329-343.

Gospodarowicz, D., and J. Cheung (1986) Heparin protects basic and acidic FGF from inactivation. J. Cell Physiol. 128: 475-484.

Gospodarowicz, D., G. Neufeld, and L. Schweigerer (1986) Molecular and biological characterization of fibroblast growth factor, an angiogenic factor which also controls the proliferation and differentiation of mesoderm and neuroectoderm derived cells. Cell. Diff. 19: 1-17.

Greene, L. A., and E. M. Shooter (1980) The nerve growth factor. Annu. Rev. Neurosci. 4: 353-402.

Grynkiewicz, G. M., M. Poenie, and T. Y. Tsien (1985) A new generation of $\mathrm{Ca}$ indicators with greatly improved fluorescence properties. J. Biol. Chem. 260: 3440-3450.

Gundersen, R. W., and J. N. Barrett (1980) Characterization of the turning response of dorsal root neurites toward nerve growth factor. J. Cell Biol. 87: 546-554.

Hatten, M. E., M. Lynch, R. E. Rydel, J. Sanchez, J. Joseph-Silverstein, D. Moscatelli, and D. B. Rifkin (1988) In vitro neurite extension by granule neurons is dependent upon astroglial-derived fibroblast growth factor. Dev. Biol. 125: 280-289.

Hauser, K. F., P. J. McLaughlin, and I. S. Zagon (1987) Endogenous opiods regulate dendritic growth and spine formation in developing rat brain. Brain Res. 416: 157-161.

Haydon, P. G., D. P. McCobb, and S. B. Kater (1984) Serotonin selectively inhibits growth cone dynamics and synaptogenesis of specific identified neurons of Helisoma. Science 226: 561-564.

Hefti, F., A. Dravid, and J. Hartikka (1984) Chronic intraventricular injections of nerve growth factor elevate hippocampal choline acetyltransferase activity in adult rats with partial septo-hippocampal lesions. Brain Res. 293: 305-311.

Hoffmann, S. R., N. W. Kowall, and A. C. McKee (1988) Calbindin D28 neurons in the hippocampal formation are resistant to degeneration and do not develop regenerative features in Alzheimer's disease. Soc. Neurosci. Abstr. 14: 154.

Hume, R. I., L. W. Role, and G. D. Fischbach (1983) Acetylcholine release from growth cones detected with patches of acctylcholine receptor-rich membranes. Nature 305: 632-634.

Kater, S. B., M. P. Mattson, C. S. Cohan, and J. A. Connor (1988) Calcium regulation of the neuronal growth cone. Trends Neurosci. 11: 315-321.
Kater, S. B., P. B. Guthrie, and S. B. Kater (1989) Calcium-induced neuronal degeneration: A normal growth cone regulating signal gone awry? Ann. NY Acad. Sci. (in press).

Koh, J., and D. W. Choi (1988) Cultured striatal neurons containing NADPH-diaphorase or acetylcholinesterase are selectively resistant to injury by NMDA receptor agonists. Brain Res. 446: 374-378.

Kordower, J. H., D. M. Gash, M. A. Bothwell, L. B. Hersh, and E. J. Mufson (1988) Nerve growth factor receptor immunoreactivity in the human and monkey brain: Distribution and colocalization with cholinergic enzymes. Soc. Neurosci. Abstr. 14: 1222.

Korsching, S. (1986) The role of nerve growth factor in the CNS. Trends Neurosci. 9: 570-573.

Korsching, S., G. Auberger, R. Heumann, J. Scott, and H. Theonen (1985) Levels of nerve growth factor and its mRNA in the central nervous system of the rat correlates with cholinergic inncrvation. EMBO J. 4: 1389-1393.

Lankford, K. L., F. G. DeMello, and W. L. Klein (1987) A transient embryonic dopamine receptor inhibits growth cone motility and neurite outgrowth in a subset of avian retina neurons. Neurosci. Lett. 75: $169-174$.

Lauder, J. M. (1988) Neurotransmitters as morphogens. Prog. Brain Res. 73: 365-386.

Leonard, D. G. B., E. B. Ziff, and L. A. Greene (1987) Identification and characterization of mRNAs regulated by nerve growth factor in PC1 2 cells. Mol. Cell. Biol. 9: 3156-3167.

Levi-Montalcini, R. (1976) The nerve growth factor: Its role in growth, differentiation and function of the sympathetic adrenergic neuron. Prog. Brain Res. 45: 235-258.

Lewis, D., D. Rausch, L. Eiden, and J. Barker (1988) Calcium currents exhibit altered sensitivity to BAY K8644 in PC12 cells differentiated with NGF and V-SRF. Soc. Neurosci. Abstr. 14: 137.

Lipton, S. A., and S. B. Kater (1989) Neurotransmitters in neuronal outgrowth, plasticity and degeneration. Trends Neurosci. (in press).

Lipton, S. A., M. P. Frosch, M. D. Phillips, D. L. Tauck, and E. Aizenman (1988) Nicotinic antagonists enhance process outgrowth by rat retinal ganglion cells in culture. Science 239: 1293-1296.

Logan, A. (1988) Elevation of acidic fibroblast growth factor mRNA in lesioned rat brain. Mol. Cell. Endocrinol. 58: 275-278.

Lynch, G. (1986) Synapses, Circuits, and the Beginnings of Memory, MIT Press, Cambridge, MA.

Maragos, W. F., J. T. Greenamyre, J. B. Penney, and A. B. Young (1987) Glutamate dysfunction in Alzheimer's disease: An hypothesis. Trends Ncurosci. 10:65-68.

Martin, D. P., R. E. Schmidt, P. S. DiStefano, O. H. Lowry, J. G. Carter, and E. M. Johnson (1988) Inhibitors of protein synthesis and RNA synthesis prevent neuronal death caused by nerve growth factor deprivation. J. Cell Biol. 106: 829-844.

Mattson, M. P. (1988) Neurotransmitters in the regulation of neuronal cytoarchitecture. Brain Res. Rev. 13: 179-212.

Mattson, M. P. (1989) Cellular signaling mechanisms common to the development and degeneration of neuroarchitecture. Mech. Ageing Dev. (in press).

Mattson, M. P., and S. B. Kater (1987) Calcium regulation of neurite elongation and growth cone motility. J. Neurosci. 7: 4034-4043.

Mattson, M. P., and S. B. Kater (1988) Isolated hippocampal neurons in cryopreserved long term culture: Development of neuroarchitecture and sensitivity to NMDA. Int. J. Dev. Neurosci. 6: 439-452.

Mattson, M. P., and S. B. Kater (1989a) Excitatory and inhibitory neurotransmitters in the generation and degeneration of hippocampal neuroarchitecture. Brain Res. 478: 337-348.

Mattson, M. P., and S. B. Kater (1989b) Development and selective neurodegencration in cell cultures from different hippocampal rcgions. Brain Res. 490: 110-125.

Mattson, M. P., P. Dou, and S. B. Kater (1988a) Outgrowth-regulating actions of glutamate in isolated hippocampal pyramidal neurons. J. Neurosci. 8: 2087-2100.

Mattson, M. P., R. E. Lee, M. E. Adams, P. B. Guthrie, and S. B. Kater (1988b) Interactions between entorhinal axons and target hippocampal neurons: A role for glutamate in the development of hippocampal circuitry. Neuron 1: 865-876.

Mattson, M. P., P. B. Guthrie, and S. B. Kater (1988c) Intracellular messengers in the generation and degeneration of hippocampal neuroarchitecture. J. Neurosci. Res. 21: 447-464.

Mattson, M. P., P. B. Guthrie, B. C. Hayes, and S. B. Kater (1989) 
Roles for mitotic history in the generation and degeneration of neuroarchitecture. J. Neurosci. 9: 1223-1232.

McCobb, D. P., P. G. Haydon, and S. B. Kater (1987) Dopamine and serotonin regulation of neurite outgrowth in different identified neurons. J. Neurosci. Res. 19: 19-26.

McDonald, J. W., F. S. Silverstein, and M. V. Johnston (1987) MK801 protects the neonatal brain from hypoxic-ischemic damage. Eur. J. Pharmacol. 140: 359-361.

Mizel, S. B., and J. R. Bamburg (1976) Studies on the action of nerve growth factor. III. Role of protein synthesis in the process of neurite outgrowth. Dev. Biol. 49: 20-28.

Morrison, R. S., A. Sharma, J. De Vellis, and R. A. Bradshaw (1986) Basic fibroblast growth factor supports the survival of cerebral cortical neurons in primary culture. Proc. Natl. Acad. Sci. USA 83: 75377541.

Needels, D. L., and C. W. Cotman (1988) Basic fibroblast growth factor increases the survival of rat dentate granule cells in culture. Soc. Neurosci. Abstr. 14: 363.

Oppenheim, R. W. (1987) Muscle activity and motor neuron death in the spinal cord of the chick embryo. In Selective Neuronal Death, G. Bock and M. O'Connor, eds., pp. 96-112, Wiley, New York.

Pearce, I. A., M. A. Cambray-Deakin, and R. D. Burgoyne (1987) Glutamate acting on NMDA receptors stimulates neurite outgrowth from cerebellar granule cells. FEBS Lett. 223: 143-147.

Purves, D. (1986) The trophic theory of neural connections. Trends Neurosci. 9: 486-489.

Rothman, S. M., and J. W. Olney (1986) Glutamate and the pathophysiology of hypoxic-ischemic brain damage. Ann. Neurol. 19: 105111.

Saji, M., and D. J. Reis (1987) Delayed transneuronal death of substantia nigra neurons prevented by GABA agonist. Science 235: 6669.

Schubert, D., M. LaCorbiere, C. Whitlock, and W. Stallcup (1978) Alterations in the surface properties of cells responsive to nerve growth factor. Nature 273: 718-723.

Schwarcz, R., A. C. Foster, E. D. French, W. O. Whetsell, and C. Kohler (1984) Excitotoxic models for neurodegenerative disorders. Life Sci. 35: 19-32.

Shelton, D. L., and L. F. Reichardt (1986) Studies on the expression of the B nerve growth factor (NGF) gene in the central nervous system: Level and regional distribution of NGF mRNA suggest that NGF functions as a trophic factor for several distinct populations of neurons. Proc. Natl. Acad. Sci. USA 83: 2714-2718.

Siesjo, B. K. (1988) Historical overview: Calcium, ischemia and death of brain cells. Ann. NY Acad. Sci. 522: 638-661.

Siman, R., and J. C. Noszek (1988) Excitatory amino acids activate calpain I and induce structural protein breakdown in vivo. Neuron 1: $279-287$

Simon, R. P., J. H. Swan, T. Griffiths, and B. S. Meldrum (1984) Blockade of N-methyl-D-aspartate receptors may protect against ischemic damage in the brain. Science 226: 850-852.

Sloviter, R. S. (1983) "Epileptic" brain damage in rats induced by sustained electrical stimulation of the perforant path. I. Acute electrophysiological and light microscopic studies. Brain Res. Bull. 10: 675-697.

Streit, J., and H. D. Lux (1987) Voltage dependent calcium currents in PC12 growth cones and cells during NGF-induced cell growth Pfluegers Arch. 408: 634-641.

Tulipan, N., S. Luo, G. S. Allen, and W. O. Whetsell (1988) Striatal grafts provided sustained protection from kainic and quinolinic acidinduced damage. Exp. Neurol. 102: 325-332.

Unsicker, K., H. Reichert-Preibxch, R. Schmidt, B. Pettman, G. Labourdette, and M. Sensebrenner (1987) Astroglial and fibroblast growth factors have neurotrophic functions for cultured peripheral and central nervous system neurons. Proc. Natl. Acad. Sci. USA 84 5459-5463.

VanHoesen, G. W., B. T. Hyman, and A. R. Damasio (1986) Cellspecific pathology in neural systems of the temporal lobe in $\mathrm{Alz}$ heimer's disease. Prog. Brain Res. 70: 321-335.

Varon, S., B. Pettmann, and M. Manthorpe (1988) Humoral and surface-anchored factors in development and repair of the nervous system. Prog. Brain Res. 73: 465-489.

Walicke, P. A. (1988) Basic and acidic fibroblast growth factors have trophic effects on neurons from multiple CNS regions. J. Neurosci. 8: 2618-2627.

Walicke, P., W. M. Cowan, N. Ueno, A. Baird, and R. Guillemin (1986) Fibroblast growth factor promotes survival of dissociated hippocampal neurons and promotes neurite extension. Proc. Natl. Acad. Sci. USA 83: 3012-3016.

Walicke, P. A., J. J. Feiges, and A. Baird (1989) Characterization of the neuronal receptor for basic fibroblast growth factor. J. Biol. Chem. (in press).

Whetsell, W. O., Jr., G. S. Allen, and N. B. Tulipan (1989) Alteration of kainic acid and quinolinic acid toxicity by neostriatal transplants in vitro. Neurosci. Lett. 96: 18-22.

Whittemore, S. R., P. L. Friedman, D. Larhammar, H. Persson, M. Gonzalez-Carvajal, and V. R. Holets (1988) Rat $\beta$-nerve growth factor sequence and site of synthesis in the adult hippocampus. $J$. Neurosci. Res. 20: 403-410.

Young, S. H., and M. M. Poo (1983) Spontaneous release of transmitter from growth cones of embryonic neurons. Nature 305: 634 637. 